\title{
A Proposal To Improve SEP Routing Protocol USINg INSENSITIVE FuZZY C-MEANS IN WIRELESS SENSOR NETWORK
}

\author{
Phan Thi The, Ngo Quang Quyen, Vu Ngoc Phan and Tran Cong Hung \\ Post \& Telecommunications Institute of Technology, Vietnam
}

\begin{abstract}
Nowadays, with the rapid development of science and technology and the ever-increasing demand in every field, wireless sensor networks are emerging as a necessary scientific achievement to meet the demand of human in modern society. The wireless sensor network (WSN) is designed to help us not lose too much energy, workforce, avoid danger and they bring high efficiency to work. Various routing protocols are being used to increase the energy efficiency of the network, with two distinct types of protocols, homogenous and heterogeneous. In these two protocols, the SEP (Stable Election Protocol) is one of the most effective heterogeneous protocols which increase the stability of the network. In this paper, we propose an approaching the EFCM algorithm in clustering the SEP protocol which makes the WSN network more energy efficient. The simulation results showed that the SEP-EFCM proposed protocol performed better than the conventional SEP protocol
\end{abstract}

\section{KEYWORDS}

Wireless sensor network (WSN), Insensitive Fuzzy C-Means, Stable Election Protocol(SEP), SEPInsensitive Fuzzy C-Means.

\section{INTRODUCTION}

The WSN is a network of interconnected sensor nodes. Sensor nodes are designed compactly and low cost. The nodes are responsible for sensing ambient conditions such as temperature, sound, vibration, humidity, pressure, etc. Sensor nodes send their sensed data to the aggregation node and transmit data to Sink (transceiver). As shown in Figure 1, Sink is transmitted over the internet or satellite to the user. In this process, we see that the $\mathrm{CH}$ (Cluster Head) not only listen to the signals from the nodes not also synthesize data, then transmit data on Sink so that more energy is consumed, so the routing, specifying the path of this data stream is very important.

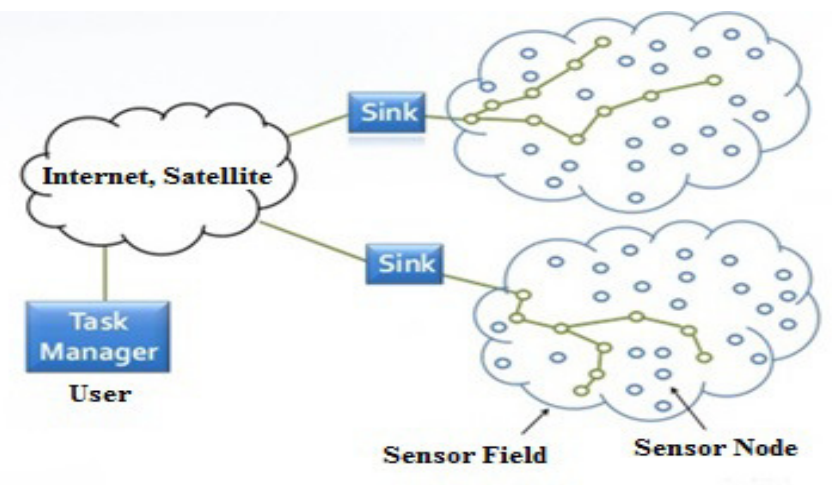

Firgure 1: The basic structure of wireless sensor networks

DOI: $10.5121 / \mathrm{ijcnc} .2017 .9604$ 
The routing protocols are divided into two types that are homogeneous and heterogeneous. Homogeneous routing protocols are nodes with the same energy level as the protocols: LEACH, TEEN, HEED, PEGASIS, APTEEN. The heterogeneous protocol is a protocol where nodes have different energy levels and are divided into two node types: the Advance node and the Normal node. In that, the Advance node has more energy and the probability of becoming a node is more than the remaining node. Popular protocols in this type of protocol are SEP[1-5], DEEC, EDEEC. SEP protocol consider the energy levels in the CH selection process and improves the stability of the hierarchical clustering process using characteristic parameters of heterogeneity, adding energy between the advance node and the normal node. In order to prolong the stabilization time, SEP strives to maintain limited energy consumption. The advance nodes will become $\mathrm{CH}$ more than normal nodes and will be powered more than normal nodes. However, the selection of Cluster Head in the SEP protocol has a disadvantage which from the two types of node Advance node and Normal node is not flexible, therefore, that remote nodes will die first. To solve this problem, We conducted the our research about SEP and evaluated some well-known clustering algorithms such as K-Means, Fuzzy CMeans, Insensitive Fuzzy C-Means in WSN clustering. Based on the learning theory, We propose a new approach which combines the Insensitive Fuzzy C-Means algorithm into the SEP protocol which can make the WSN network more energy efficient. Additionally, we used Matlab to simulate for the new algorithm, for 140 nodes in a network 500x500, with uneven energy between nodes to show unequal influence among nodes in the network. $10 \%$ of the nodes have 1 Joule energy and $90 \%$ of nodes have 0.5 Joules energy. The position of the sink is set at $(250,250)$ and the length of each message is 500bytes. The simulation results show that the proposed SEP-eFCM protocol performed better than the conventional SEP protocol.

Our paper consists of five parts: Part 1 is introduction, Part 2 shows the related works, Part 3 proposal, Part 4 simulation results and Final Part is the conclusion.

\section{RELATED WORK}

If the LEACH was Malik M.et al. [6] gave as a basic protocol for routing and values that are effective for WSN networks, Smaragdakis has introduced a revolutionary SEP routing protocol. SEP is a heterogeneous routing protocol and has proven to be more effective than the LEACH protocol. In SEP protocol, the node sensors are divided into two types of nodes: the advance node and the normal node in which the advance node is designed to have a higher energy level and the probability becomes $\mathrm{CH}$ node than the normal node. As shown in Figure 2 


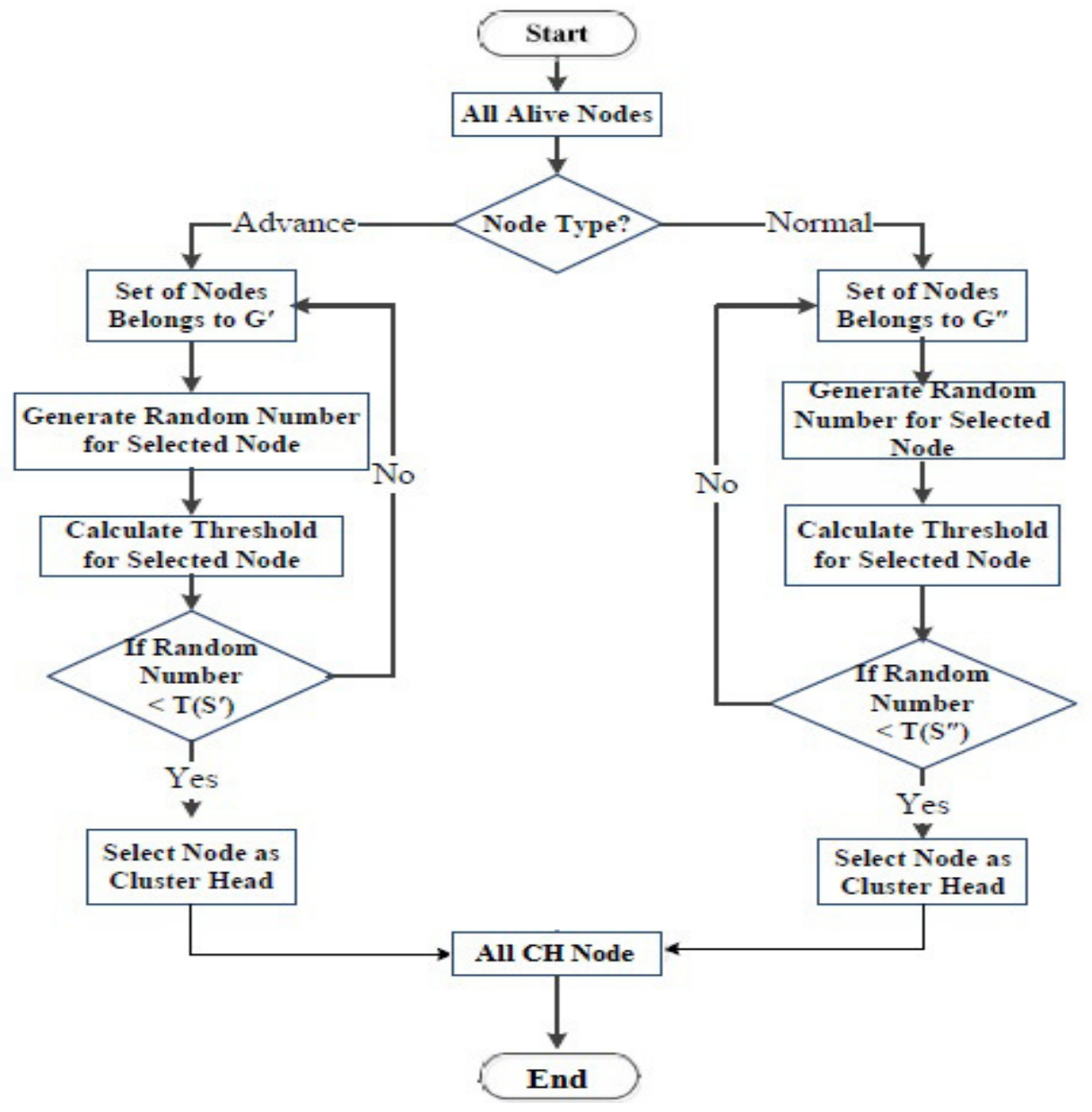

Figure 2: Flow chart of $\mathrm{CH}$ Selection in SEP protocol

Where:

- G': Normal node does not become $\mathrm{CH}$.

- $\quad \mathrm{T}\left(\mathrm{S}^{\prime}\right)$ is the threshold applied to normal node.

- G”: advanced node does not become CH.

- $\quad \mathrm{T}\left(\mathrm{S}^{\prime \prime}\right)$ is the threshold applied to advanced node.

If the K-Means algorithm [5] is a well-defined clustering algorithm, it means that one node belongs only to one cluster, which is only suitable for discovering high density and isolated clusters and must define the boundary. However, in practice when deploying WSN, the boundary between clusters may be blurry, clusters may overlap, meaning that some nodes belong to different clusters. So with this case, using the K-means algorithm will limit the functionality of the wireless sensor network, so the Fuzzy c-means (FCM) algorithm [7-9] was born to solve this problem. FCM is a fuzzy clustering algorithm developed by Dunn in 1973 and later improved by Bezděk in 1981. This technique divides a set of n-vector data objects $X=\{x 1, \ldots, x n\} \in$ Rs into the fuzzy groups which are based on the minimization of the objective function to measure the quality of the partition and find the cluster centers in each cluster, so that the cost of the nonsimilarity measure is minimal. 
By 1998 Vapnick proposed using the parameter $\varepsilon$ is called a noise-sensitive parameter to resolve high-density clusters that overlap, where one node is not only attached to a cluster but can belong to one cluster or many different clusters. Since then, $\varepsilon$ FCM[10-14] has always been a top choice for fuzzy clustering.

In this article the simulated results show that the selection of $\mathrm{CH}$ node by $\mathrm{EFCM}$ algorithm reduces the energy consumption of the WSN.

\section{Proposed Algorithm}

Heterogeneous protocol SEP has been proven by Smaragdakis's to provide stability and longer lifetimes than unified protocols. However, they still contain a number of limitations, including limited clustering for high node density clusters and complex multi-dimensional distribution, clustering that leads to uneven clusters. (Less node clusters and multiple node clusters) that cause network load balancing.

In this paper, We proposes using the $\mathrm{EFCM}$ algorithm to select $\mathrm{CH}$ nodes for the SEP protocol, and selecting this node initially gives priority to the nodal node to take advantage of this additional energy source as $\mathrm{CH}$ Node to create the most stable time for the network. After the advance nodes consume the remaining energy and energy equal to the normal node, then $\varepsilon F C M$ will proceed to select $\mathrm{CH}$ node over the entire network, preferring that node which has more energy will be made $\mathrm{CH}$ node. The target function of the $\varepsilon \mathrm{FCM}$ algorithm is defined as follows:

$$
J_{m \varepsilon}(U, V)=\sum_{k=1}^{n} \sum_{i=1}^{c}\left(u_{i k}\right)^{m}\left\|x_{k}, v_{i}\right\|_{\varepsilon}
$$

Where $\left\|x_{k}, v_{i}\right\|_{\varepsilon}=\sum_{l=1}^{p}\left\|x_{k l}-v_{p}\right\|_{\varepsilon}$, $\varepsilon$ is non-sensitive to noise are Vapnick proposed in 1998 as follows::

$$
\begin{gathered}
\|t\|_{\varepsilon}= \begin{cases}0, & \|t\| \leq \varepsilon \\
\|t\|-\varepsilon & \|t\|>\varepsilon\end{cases} \\
u_{i k}=\left\{\begin{array}{l}
\frac{1}{\sum_{j=1}^{c}\left(\frac{d_{i k}}{d_{j k}}\right)^{\frac{2}{m-1}} ; 1 \leq i \leq c, 1 \leq k \leq n} \\
\left\{\begin{array}{l}
0 ; i \neq I_{k} \\
\sum_{i \in I_{k}} u_{i k}=1 ; \quad i \neq I_{k}, I_{k} \neq \emptyset
\end{array}\right. \\
\forall_{1 \leq i \leq c} \forall_{1 \leq l \leq p} v_{i l}=\frac{1}{\operatorname{card}\left(A_{i}^{+} \cup\right) A_{i}^{-}}\left[\sum_{\left\{k \mid \lambda_{k}^{+} \in A_{i}^{+}\right\}}\left(x_{k l}+\varepsilon\right)+\sum_{\left\{k \mid \lambda_{k}^{-} \in A_{i}^{-}\right\}}\left(x_{k l}-\varepsilon\right)\right]
\end{array}\right.
\end{gathered}
$$

\section{The steps of the \&FCM algorithm:}

Input: The number of clusters $\mathrm{c}$ and the parameter $\mathrm{m}, \varepsilon$ for the objective function $\mathrm{J}$;

Output: The cluster data so that the objective function (1) reaches the minimum value;

\section{Begin}

1. Input parameter $\mathrm{c}(1<\mathrm{c}<n), \mathrm{m}(1<\mathrm{m}<+\infty)$ and $\varepsilon \geq 0$; Initialize the matrix $\mathrm{V}=\left[v_{i j}\right], \mathrm{V}^{(0)} \in R^{s x c}$, set $\mathrm{j}=0$; 
2. Repeat

$2.1 \mathrm{~J}:=\mathrm{j}+1$;

2.2 Calculate fuzzy partition matrix $\mathrm{U}^{(\mathrm{j})}$ according to the formula (2);

2.3 Update center clusters $\mathrm{V}^{(\mathrm{j})}=\left[v_{1}^{(j)}, v_{2}^{(j)}, \ldots \ldots, v_{c}^{(j)}\right]$ according to the formula (3) and $\mathrm{U}^{(\mathrm{j})}$

3. Until $\left(\left\|U^{(j+1)}-U^{j}\right\|_{F} \leq \varepsilon\right)$;

End

4. Demonstrate result of clusters;

The SEP protocol [10-12] takes into account the energy level in the main node selection process. SEP improves the stability of the hierarchical clustering process using the characteristic parameters of the heterogeneous, additional energy between the advanced node and the normal node. To prolong the stabilization time, SEP strives to maintain its energy consumption limit. Advanced nodes become $\mathrm{CH}$ more often than normal nodes. Advanced nodes usually have more power than the normal node. The total energy of the system varies. Assuming Eo is the initial energy of the normal node, the energy of the advanced node is set to Eo * $(1+\alpha)$. The total energy needed to set up (new) is equivalent to: $n *(1-m) *$ Eo $+n * m *$ Eo $(1+\alpha)=n *$ Eo $(1+$ $\alpha \mathrm{m})$. Thus the total energy of the system is increased $(1+\alpha \mathrm{m})$ times. We can increase the stability of the sensor network $(1+\alpha \mathrm{m})$ times.

The probability that the normal node becomes $\mathrm{CH}$ is 1 and the advanced node becomes $\mathrm{CH}$ is $1+$ $\alpha$. If the threshold $\mathrm{T}(\mathrm{n})$ is set to normal and the advanced node differs in that the normal node of $\mathrm{G}$ becomes the first cluster once times and the advanced node of $\mathrm{G}$ becomes the first cluster $1+\alpha$ times. Pnrm is defined as the probability of selecting weights for normal nodes and the probability of selecting weights for advanced nodes. So the weight probabilities for normal node and advanced node nodes are:

$$
\begin{aligned}
& \text { Pnrm }=\frac{\text { Popt }}{1+\alpha m} \\
& \text { Padv }=\frac{\text { Popt }}{1+\alpha m} x(1+\alpha)
\end{aligned}
$$

The function T (n) is replaced by Popt by the weight probability to obtain the selection threshold $\mathrm{CH}$ in each round.

- The threshold for normal node:

Where:

$$
\mathrm{T}(\text { Snrm })=\left\{\begin{array}{lr}
\frac{\text { Pnrm }}{1-\text { Pnrm }\left(r * \text { mod } \frac{1}{\text { Pnrm }}\right)} \text { if } & \text { Snrm } \in \text { G }, \\
0 & \text { another case }
\end{array}\right.
$$

- $\quad$ r: current loop

- G': Normal node does not become CH with 1 / Pnrm last cycle per phase.

- $\quad \mathrm{T}(\mathrm{Snrm})$ is the threshold applied to $\mathrm{n}(1-\mathrm{m})$ normal node. This ensures that each normal node becomes $\mathrm{CH}$ once time in $\frac{1}{\text { Popt }}(1+\alpha \mathrm{m})$ per phase and that is the average of the normal nodes becoming $\mathrm{CH}$ each round is $\mathrm{n}(1-\mathrm{m}) *$ Pnrm

- The Threshold for advanced node 


$$
\mathrm{T}(\operatorname{Sadv})= \begin{cases}\frac{P a d v}{1-\text { Padv }\left(r * \bmod \frac{1}{\text { Padv }}\right)} & \text { if Snrm } \in \mathrm{G}, \\ 0 & \text { another case }\end{cases}
$$

Where

- G”: advanced node does not become $\mathrm{CH}$ CH in $\frac{1}{P a d v}$ last cycle per phase.

- $\quad \mathrm{T}(\mathrm{Sadv})$ is the threshold applied to $\mathrm{n} * \mathrm{~m}$ advanced node.

We consider to this phase as the secondary stage. Each stage has $1+\alpha$ sub-stage and the advanced node becomes $\mathrm{CH}$ exactly $1+\alpha$ times in phase. The average of advanced node becomes $\mathrm{CH}$ for per cycle which has $\mathrm{n} *$ Padv.

So the average number of CHs per round is: $\mathrm{n} *(1-\mathrm{m}) * \operatorname{Pnrm}+\mathrm{n} * \mathrm{~m} * \mathrm{Padv}=\mathrm{n} *$ Popt. This is the desired number of $\mathrm{CHs}$ in per phase.

\section{Simulation And Evaluation}

In this paper, We uses Matlab software (R2016a) to perform simulations, for 140 nodes which have fixed coordinates in a network 500x500, with uneven energy between nodes to show unequal influence among nodes in the network. $10 \%$ of nodes have 1 Joules energy $(\mathrm{a}=1$, Popt $=$ $0.1), 90 \%$ of nodes have 0.5 Joules energy. The position of the sink is set at $(250,250)$, the length of each message is 500bytes, the amplification coefficient efs $=10 \mathrm{pJ} /$ bit $/ \mathrm{m} 2$ and e mp $=0.0013 \mathrm{pJ} / \mathrm{bit} / \mathrm{m} 4$, the maximum number of loops is 6000.The input parameters are fixed, the author will in turn put these parameters into running SEP protocol and proposed protocol SEP $\varepsilon F C M$. Then we compare between protocol combining SEP and SEP_ $\varepsilon F C M$ protocol based on metrics such as: number of live nodes, number of Dead nodes and the remaining energy of nodes.

\begin{tabular}{|l|l|}
\hline \multicolumn{1}{|c|}{ PARAMETERS } & \multicolumn{1}{c|}{ VALUE } \\
\hline Network size & $500 \times 500$ \\
\hline BS & $(250,250)$ \\
\hline Rounds & 6000 \\
\hline Iteration & 200 \\
\hline$\square \mathrm{fs}$ & $10 \mathrm{pJ} / \mathrm{bit} / \mathrm{m}^{2}$ \\
\hline$\square \mathrm{mp}$ & $0.0013 \mathrm{pJ} / \mathrm{bit} / \mathrm{m}^{4}$ \\
\hline EDA & $5 \mathrm{~nJ} / \mathrm{bi} / \mathrm{packet}$ \\
\hline E 0 & $0.5 \mathrm{~J}$ \\
\hline N & 140 \\
\hline A & 1 \\
\hline K & 15 \\
\hline Popt & 0.1 \\
\hline
\end{tabular}




\subsection{Results AfTer The First 1200 RoundS}

In Figure 3, on the SEP protocol we see that after 1200 cycles the dead node has appeared, while in the SEP $\_$FCM association we see the result after 1200 cycles in Figure 4 shows no dead node.

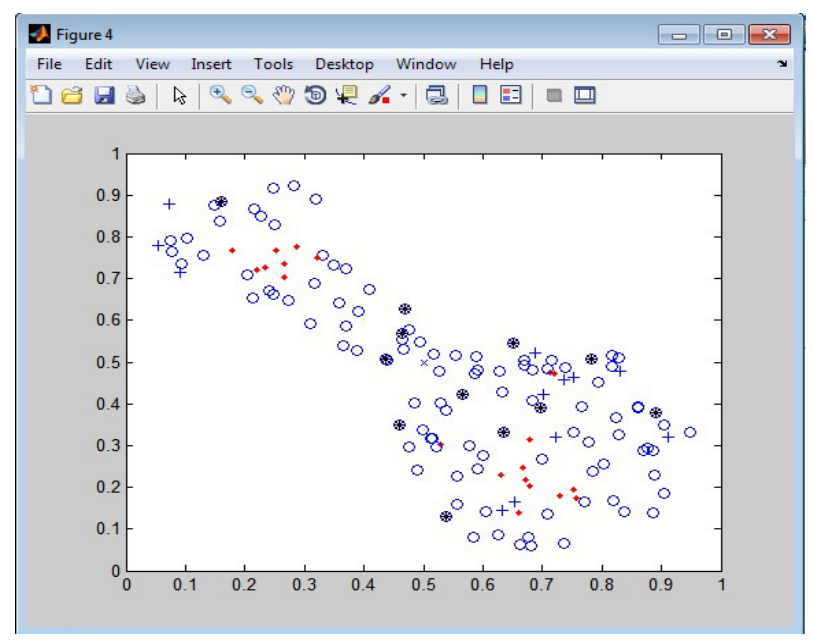

Figure 3: SEP protocol after 1200 rounds

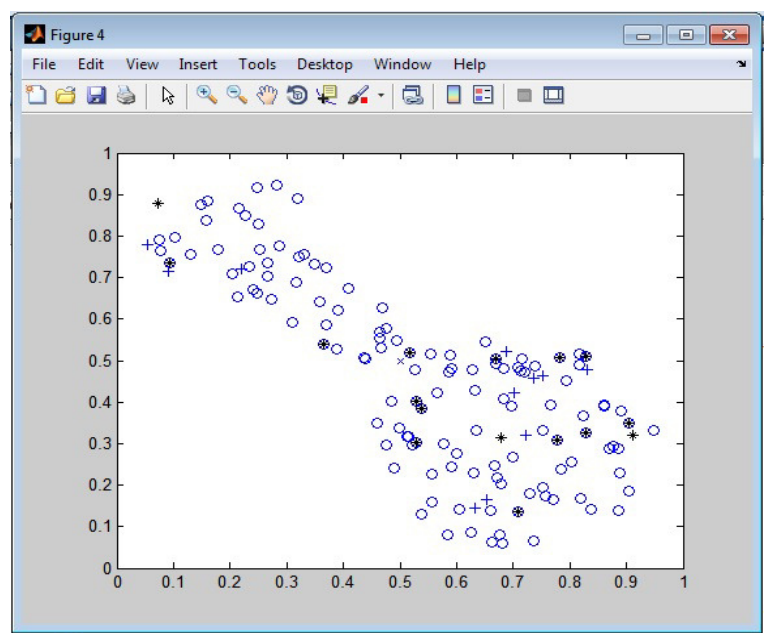

Figure 4: SEP_ $\varepsilon F C M$ association protocol after 1200 rounds

\subsection{NUMber OF LiVE NODES}

Figure 5 show the number of alive during the network lifetime. After 800 cycles, SEP appears as the first dead node, after 2790 SEP_ $\varepsilon F C M$ loops appear. Proposed protocol is higher $21 \%$ SEP of the number of rounds that the last node dies.

Therefore, the living nodes in the SEP_ $\varepsilon F C M$ combination protocol have more live nodes than the SEP protocol. After a few initial rounds are unstable, but then the number of live nodes starts to increase, this proves that the $\mathrm{SEP}_{-} \varepsilon \mathrm{FCM}$ combination protocol consumes more efficient 
energy than the SEP protocol. Results for the SEP $\_$EFCM combination protocol have improved more live nodes than the SEP protocol.

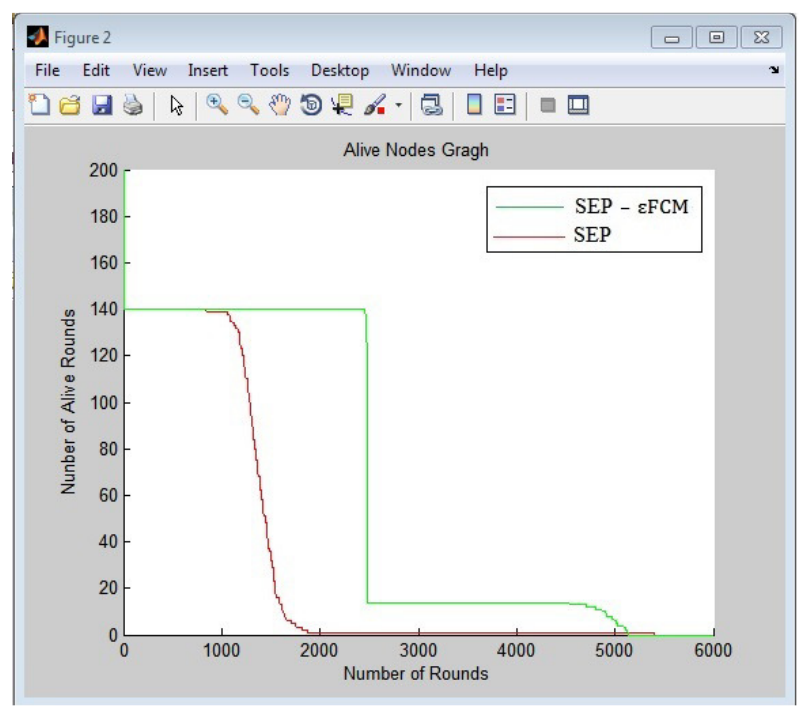

Figure 5: Alive Node comparison between the SEP protocol and SEP $\_$EFCM protocol

\subsection{REMAINING ENERGY}

In Figure 6 we find that, after 4985 round run, the node in SEP died out and 5150 SEP $\varepsilon F C M$ nodes. Proposed protocol is higher 3.3\% SEP. The result shows that the Remaining energy of the $\mathrm{SEP}_{-} \varepsilon \mathrm{FCM}$ matching protocol is greater than that of the SEP protocol. The results of the SEP $\varepsilon$ FCM combination protocol have improved the residual energy of the SEP protocol.

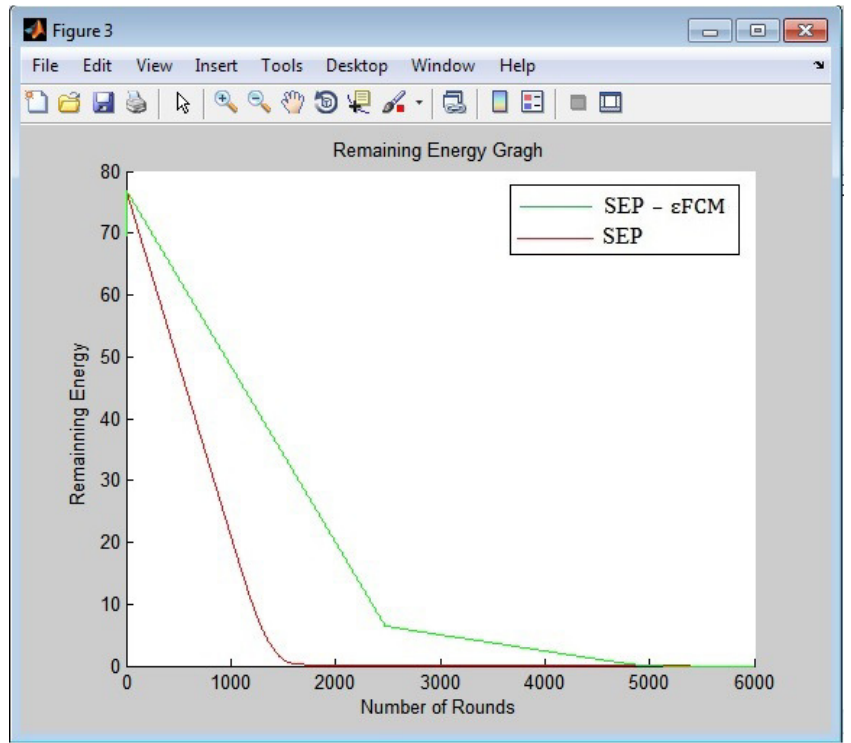

Figure 6: Remaining energy comparison between the SEP protocol and SEP_ $\_F C M$ protocol 


\section{Conclusions}

Designing WSN network with well-functioning, flexible and easy-to-deploy for real-world applications is fraught with many difficulties, with the biggest difficulty now being the limited node power and hard to reload. Therefore, utilizing available power resources on nodes effectively reduces the energy consumption that extends the life of the entire network, which increases the life of the network.We researched the routing protocols to propose the new algorithm using the $\varepsilon F C M$ fuzzy algorithm in the selection of $\mathrm{CH}$ node into the SEP protocol. Simulation results suggest that our improvement has lower power consumption and longer network lifetime than the 3.3\% SEPprotocol. This helps the network extend the lifespan.

\section{REFERENCES}

[1]. D. M. a. T. Z. K. Sohraby (2007), Wireless Sensor Network Technology, Protocol, And Application, John Wiley \& Sons, Inc.

[2]. S. Basagni (1999), Distributed Clustering Algorithm for Ad Hoc Networks, Proc. Int'l. Symp. Parallel Architectures, Algorithms, and Networks.

[3]. S. B. a. S. Khuller (Apr. 2001), A Clustering Scheme for Hierarchical Control in Multihop Wireless Networks, Proc. IEEE INFOCOM.

[4]. Srinivas Sivarathril and A.Govardhan2 (September 2014), "Experiments on Hypothesis Fuzzy KMeans is Better Than K-Means for Clustering", International Journal of Data Mining \& Knowledge Management Process (IJDKP) Vol.4, No.5.

[5]. Keerthi M, M.Tech 2nd year and Dr. B. Satish Babu,Professor (August 2012), "An Improved FCM's Clustering Protocol for Wireless Sensor Networks", International of Electronics and Communications (IJEC), Volume-1,Issue-1.

[6]. Meena Malik, Yudhvir Singh, Anshu Arora (February 2013), "Analysis of LEACH Protocol in Wireless Sensor Networks", International Journal of Advanced Research in Computer Science and Software Engineering, Volume 3, Issue 2, pp. 178-183.

[7]. Maurad Hadjilal, Hervé Guyennet1, Mohammed Feham (2013), "Energy-Efficient in wireless sensor networks using fuzzy C-Means clustering approach", International Journal of Sensors and Sensor Network.

[8]. A. S. Raghuvanshi*, S Tiwari, R Tripathi and N. Kishor "Optimal Number of Clusters in Wireless Sensor Networks: An FCM Approach"Motilal Nehru National Institute of TechnologyAllahabad 211004, INDIA.

[9]. Raja Dutta, Shishir Gupta, Mukul K. Das (November 2014), "Low-Energy Adaptive Unequal Clustering Protocol Using Fuzzy c-Means in Wireless Sensor Networks", Wireless Personal Communications, Springer, Volume 79, Issue 2, pp. 1187-1209.

[10]. I. M. Georgios Smaragdakis, Azer Bestavros (2004), "SEP : A Stable Election Protocol for clustered heterogeneous wireless sensor networks", Computer Science Department Boston Univercity Boston, MA 02215, USA. 
[11]. G. Smaragdakis, I. Matta, A. Bestavros (2004), SEP: A Stable Election Protocol for clustered heterogeneous wireless sensor networks, in: Second International Workshop on Sensor and Actor Network Protocols and Applications (SANPA 2004).

[12]. K. Latif, M. Jaffar, N.Javaid, M. N. Saqib, U. Qasim, Z. A. Khan (2012), "Performance Analysis of Hierarchical Routing Protocols in Wireless Sensor Networks" arXiv : 1208.2397v1 [cs.NI].

[13]. Dr. Firas Ali Al-Juboori, Eng. Sura F. Ismail (2013) "Performance Analysis of Variable Energy Levels of Clustering Protocols for Wireless Sensor Network" IJCSI International Journal of Computer Science Issues, Vol.10,No 1.

[14]. Bharti Kandari, Rajdeep Singh (2014) "K-SEP: A more stable SEP using K-Means Clustering and Probabilistic Transmission in WSN" INPRESSCO International Journal of Current Engineering and Technology, Vol.4, No.4.

\section{AUTHORS}

Phan Thi The was born in Vietnam in 1982.She received Master Data Transmission and NetWork in HOCHIMINH PTIT, Vietnam, 2012. She is currently a PhD.Candidate in Information System from Post \& Telecommunications Institute of Technology, Vietnam in 2019.She is working as a lecture in Thu Duc College.

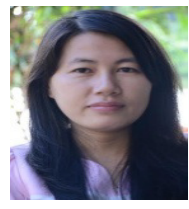

Ngo Quang Quyen was born in Vietnam in 1985. He received the B.E in Industrial Univercity of HoChiMinh city, Vietnam, 2012. He is currently a MSc. Candidate in Information System from Post \& Telecommunications Institute of Technology, Vietnam in 2017. He is working in Children's Hospital No.2.

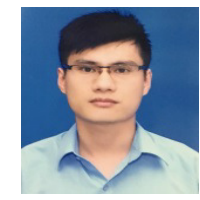

Tran Cong Hung was born in Vietnam in 1961. He received the B.E in electronic and Telecommunication engineering with first class honors from HOCHIMINH University of technology in Vietnam, 1987. He received the B.E in informatics and computer engineering from HOCHIMINH University of technology in Vietnam, 1995. He received the master of engineering degree in telecommunications engineering course from postgraduate department Hanoi University of technology in Vietnam, 1998. He received Ph.D at Hanoi University of technology in Vietnam, 2004. His main research

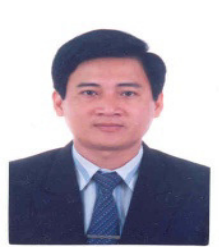
areas are B - ISDN performance parameters and measuring methods, QoS in high speed networks, MPLS. $\mathrm{He}$ is, currently, Associate Professor PhD. of Faculty of Information Technology II, Posts and Telecoms Institute of Technology in HOCHIMINH, Vietnam. 